\title{
Investigating the Role of Technical and Process Quality in Chatbots: A Case Study from the Insurance Industry
}

\author{
Tommi Pirilä \\ KPMG \\ tommi.p.pirila@gmail.com
}

Vignesh Yoganathan

University of Sheffield

v.yoganathan@sheffield.ac.uk

\author{
Joni Salminen \\ Qatar Computing Research Institute, \\ Hamad Bin Khalifa University \\ jsalminen@hbku.edu.qa \\ Bernard J. Jansen \\ Qatar Computing Research Institute, \\ Hamad Bin Khalifa University \\ bjansen@hbku.edu.qa
}

\author{
Victoria-Sophie Osburg \\ Montpellier Business School \\ vs.osburg@montpellier-bs.com
}

\begin{abstract}
Though artificial intelligence (AI) can benefit customer service, there are also user acceptance and quality concerns. We investigate these challenges that impact AI preference and adoption in a real customer service scenario. We focus on Emma, a customer service chatbot at a large Finnish insurance company. Our analysis, based on a survey of 225 consumers using the chatbot, shows that users are reasonably satisfied with Emma, though they are generally do not prefer it to a human. Process quality, relating to "soft" aspects of interaction, is essential for strengthening the users' AI preference. The chatbot's problem-solving ability acts as a hygiene factor, which alone cannot ensure adoption. As a pleasing and useful interaction is a prerequisite for AI user experience, organizations should consider both technical and process quality when implementing chatbots for customer service.
\end{abstract}

\section{Introduction}

The digital transformation has fundamentally changed the service landscape and resulted in more significant changes than ever before. Amongst others, this can be seen in the enormous growth of IT-related services, in which information and communication technology allows organizations to communicate with their customers anywhere and anytime [15]. Another related radical change was recently caused by the implementation of artificial intelligence (AI), i.e., machines that show characteristics of human intelligence such as robots or virtual bots [14, 37, 38].

Organizations expect several benefits associated with the introduction of AI marketing [23] and to the service context. For example, AI can be more reliable compared to the human workforce and accessible without any time constraints, whilst human staff is restricted by working time and job conditions, and of course, AI is never absent due to leave or sickness. There are also other benefits of AI, such as consistency of performance and reduction of errors, which are associated with cost-effective operations [5, 7, 28]. So, the attraction of automation, particularly using AI for service scenarios, is clear for organizations looking to optimize resource utilization $[16,19]$. Therefore, the implementation of AI in the service context is progressing quickly, even to the extent that human workforces are entirely replaced by AI, as in the case of Amazon's employee-free grocery stores Amazon Go. Surprisingly, though, the facets of AI and determinants of AI adoption have not been sufficiently considered and require user-facing research [18].

Nonetheless, this research must acknowledge that a human workforce is sometimes superior to AI despite the generally positive trend surrounding AI implementation. Therefore, several challenges related to the introduction of technology to the service context must be considered [9], in addition to ethical questions [36]. For example, human workers tend to be better at adding a personal touch to service interactions and seem superior in managing challenging and complex situations. Hence, the question of who performs better in a specific situation, AI or human workers, does not have a straightforward answer. Organizations, therefore, must consider a range of criteria when they are confronted with this question.

In any case, AI implementation will only be successful given user acceptance, and AI's long-term success will depend on creating a user preference for AI instead of interactions with human staff $[20,28]$. For instance, AI implementation may result in cost savings; however, these cost savings will not be advantageous if users do not accept the AI implementation or are dissatisfied with their AI-provided service experience and consequently switch to another service provider [13, 
28]. Whilst organizations may have the option to rely on either AI or human staff in some cases, the decision has already been made in other situations. AI implementation is sometimes necessary to guarantee an organization's long-term survival. Of course, users' willingness to interact with $\mathrm{AI}$ is vital in such a scenario.

User acceptance has a long tradition of being the center of discussion in information system research and practice, also regarding the implementation of new technology. For example, theoretical frameworks such as the Technology Acceptance Model (TAM1 and TAM2) [31,32] help identify the drivers of customers' technology adoption, such as perceived usefulness and ease-of-use. Despite the availability of such frameworks, we often observe in the field that technologies are continuously optimized and that technology performance (technical quality [12]) is considered as the strong driving force of technology adoption. Indeed, technical quality, including aspects such as the problem-solving ability and speed with which the problem is solved [12], is an important driver; but other drivers of user acceptance are sometimes overlooked. Such drivers include characteristics of the user-to-AI interaction, i.e., process quality [12] - is this interaction empathetic as well as efficient, and is it enjoyable and fun as much as effective?

Beyond this, there are also specific characteristics of the service situation and the customers themselves, which may facilitate or hinder preferences for AI interaction. The interaction between the user and the situation can influence the outcome and the service experience. Thus, with this research, we want to provide an unbiased view of what makes AI successful in the real world whilst focusing on the specific case of chatbots. Such understanding will help organizations to consider the range of factors influencing the success of $\mathrm{AI}$ implementation and the creation of user preferences for $\mathrm{AI}$ interaction.

Chatbots are an application of AI, which respond to user inquiries and represent an organization's virtual customer servant. Among others, chatbots can be found on messaging sites, social media sites, and also websites [29]. For example, Facebook allowed organizations to use chatbots from 2016, facilitating organizations' interaction with their customers. As chatbots simulate the conversation of a human being, users do not necessarily realize that they are interacting with $\mathrm{AI}$ instead of a human representative of the organization. However, a user's interaction with a chatbot is not always straightforward; some chatbots have problems correctly identifying the query, and they misunderstand the customer [27]. For instance, keyword-based systems can fail in understanding semantics or correctly identifying contextual meaning, which ultimately results in service failure [35].
However, machine learning techniques, which are the technical mechanisms that give rise to the intelligent abilities of the AI-powered chatbot, are improving rapidly, which positively impacts the performance of chatbots. In this case, chatbots will derive the correct meaning of a customer inquiry, enabling them to provide an appropriate response and a seamless service experience for customers. Yet, whether we have already reached this stage is not sufficiently clear.

In the following, we will take a closer look at the (dis)advantages of AI implementation in the service context and factors that organizations can consider increasing AI preference. As a specific application, we will consider the case of chatbot implementation and its impact on customer service interactions.

\section{Related Literature}

\subsection{The Choice Between Service Channels}

The implementation of technology in the service context requires that customers accept new interaction processes as the change of interacting with technology instead of human staff is a rapid change from the user's point of view [20]. These days, users may already be used to the implementation of technology in the service context, for example, in the form of self-service technology (e.g., self-service checkouts, interactive kiosks). Although such self-service technology has gained much attention in research and practice, AI differs from these service encounters in multiple ways and, therefore, needs to be considered separately. Among others, customers' interactions with AI that utilizes 'natural language' (contrasted with computer code) result in a much more human-like service exchange than users are used to from conventional self-service technology applications such as self-service checkouts at the supermarket.

Given the lack of in-depth understanding of user interaction with $\mathrm{AI}$ in the service context, we firstly focus on what is known from service technology. Obviously, traditional customer service and service technologies have their unique strengths and weaknesses. Table 1 presents some of these characteristics, which may facilitate organizations' decision-making processes.

For instance, service technology appears as advantageous if the task is relatively easy to solve [4, 28], but, at the moment, still lags behind traditional service encounters when the task is difficult, and especially if the interaction is delicate and requires a 'human touch' (e.g., conveying emotions) [9]. 
Table 1: Strengths and Weaknesses of Traditional Customer Service and Service Technology.

\begin{tabular}{|c|c|c|}
\hline & $\begin{array}{l}\text { Traditional } \\
\text { Customer Service }\end{array}$ & $\begin{array}{l}\text { Service } \\
\text { Technology }\end{array}$ \\
\hline Strengths & $\begin{array}{l}\text { - Sociality } \\
\text { - Interaction } \\
\text { - Trust } \\
\text { - Complex } \\
\text { operations }\end{array}$ & $\begin{array}{l}\text { - Economic } \\
\text { efficiency } \\
\text { - Accessibility } \\
(24 / 7) \\
\text { - Easiness } \\
\text { - Increased control } \\
\text { - Simple } \\
\text { operations }\end{array}$ \\
\hline Weaknesses & $\begin{array}{l}\text { - Labor costs } \\
\text { - Infrastructure } \\
\text { costs } \\
\text { - Changes in } \\
\text { employee rotation } \\
\text { - Rush } \\
\text { - Quality } \\
\text { fluctuations due to } \\
\text { employee } \\
\text { differences }\end{array}$ & $\begin{array}{l}\text { - Personal service } \\
\text { - Reaction speed } \\
\text { - Emotionality } \\
\text { - Quality } \\
\text { variations due to } \\
\text { technological } \\
\text { differences }\end{array}$ \\
\hline
\end{tabular}

Nonetheless, AI's capability to show not only mechanical and analytical but also intuitive and empathetic intelligence [14] is the latest development of AI and should, therefore, enable more empathetic communication with humans in the future. Organizations need to analyze the context in which AI should be implemented to determine its strengths and weaknesses to make a fully informed decision. Independent of the general superiority of one of the two approaches, the preferences for traditional customer service as opposed to AI technology may differ between individuals; hence, access to both forms could be most beneficial [22], particularly in the transition from human interaction to interacting with AI.

After deciding to continue with the AI implementation, customer experience needs to be understood as the crucial force behind user acceptance. In this context, customers' experienced quality deserves special attention, reflecting the comprehensive evaluation of the service performance [8, 34]. Users' preference for a technology primarily depends on satisfaction with the technology interaction, which is again related to quality perceptions [25]. Satisfaction can, in turn, lead to users' willingness to continuously use the technology [2]. Most importantly, the evaluation does not only include the technical performance, i.e., the "input", but also how users perceive the entire interaction, i.e., the "process" [10, 26].

The distinction between input/perceived technical quality on the one hand, and process/perceived process quality, on the other hand, is inevitable to ensure AI success. In other words, we can also differ between the "hard" and "soft" sides of AI. Figure 1 gives an overview of important elements describing the input and process of AI implementation, which ultimately affects a range of outputs related to AI implementation [5]. Organizations are advised to consider these criteria to ensure that users perceive both as high so that an AI preference can finally be achieved. It must also be noted that the "input" characteristics may induce both a direct effect on the "output", as well as an indirect effect through influencing the process quality.

Further, it is not yet clear whether users have higher expectations about AI interaction compared to service exchanges with human staff: Some work points to higher expectations for AI [33], whereas other research suggests that users are more forgiving regarding service failures of AI compared to human staff [28]. In either case, organizations need to ensure that users perceive high quality to realize long-term success.

\subsection{Process and Technical Quality}

Perceived quality can be defined broadly as a comprehensive evaluation of the service performance [7, 25]. Grönroos [11] explains that experienced comprehensive service quality originates from two factors: technical quality that is observable from endresult and process quality, meaning how the service process has proceeded from the perspective of a customer. The former is associated with the fact that can a customer get a problem solved or are direct needs towards it fulfilled. The latter - process quality - is formed from all the service's interactions. For instance, what is the accessibility of the service, how well the customer was served and how the customer experienced benefitting from the service.

Following the previous discussion, it is essential to aim at optimizing both the technical quality of AI (chatbots in the case of our study) that users perceive and the process quality $[10,26]$. It must be noted that customers' perceptions will ultimately depend on their expectations in that they compare their expected process/technical quality with their experience. Hence, after exposure to interaction with a chatbot, users will either hold high or low perceptions of process/technical quality, depending on how well their experience of the interaction fares against their prior expectations. A favorable comparison will ultimately result in a preference for interaction with chatbots. Again, it must be noted that the perceived process quality will be influenced by the perceived technical performance of the chatbot $[10,11,26]$. For example, if a chatbot is easy to access and functions correctly, important preconditions emerge for a smooth interaction and positive customer experience. 


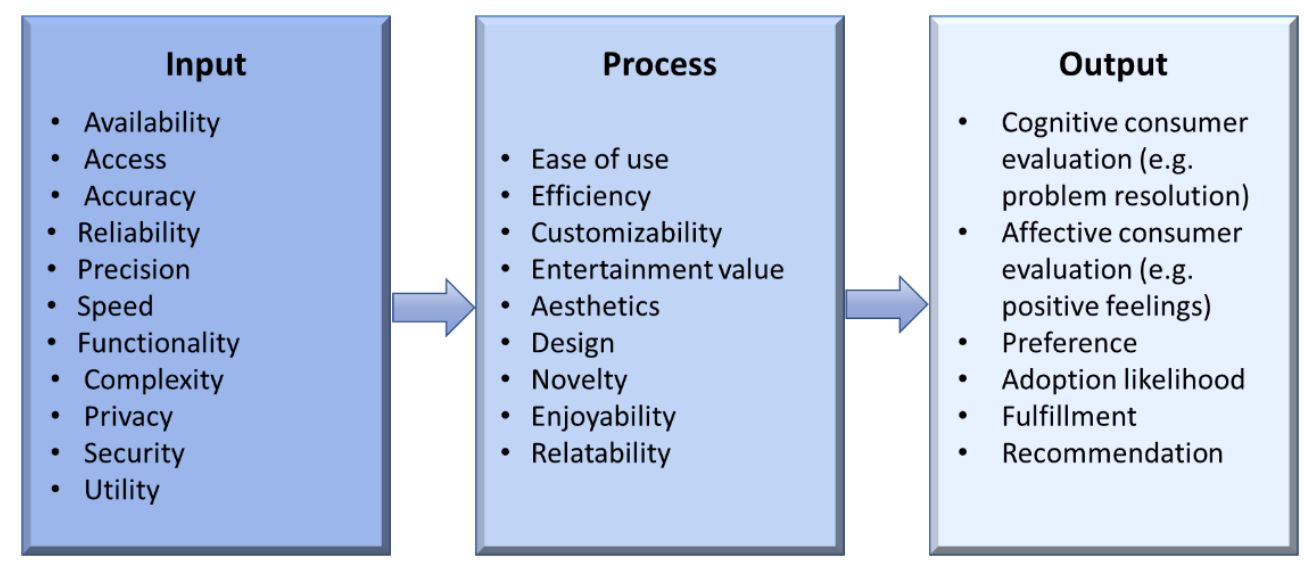

Figure 1: Input- and Process-Quality Dimensions of Service Technology.

\subsection{User Differences in the AI Preference}

Bettencourt et al. [3] argue that service innovation has long focused on mere service improvements but often neglected users' actual needs. When an organization communicates with its users, it is not only the characteristics of the communication attempt and the medium on which its success depends; the success is dependent on the characteristics of the user (e.g., their personality) and the situation (e.g., urgent or simple query). This is described by the person-situation interaction approach [24], which states that the characteristics of a situation interact with the characteristics of an individual. If both characteristics are combinable and in favor of the service encounter, it will affect the service experience positively. More specifically, if both characteristics favor the service encounter, synergistic effects may even occur. In other words, the value for users that is generated from a technologically-enabled service encounter emerges from situational and individual characteristics [20, 28].

Following the simple approach of user segmentation, AI preference may emerge from different factors for different user groups. For example, whilst process quality might be of great importance for some users, who value the interaction and personal touch of service transactions, other users, who tend to be more skeptical and consequently seek reassurance, might place special emphasis on the technical quality (e.g., security, privacy). In line with this, it has been shown that users' experienced value is affected by individual characteristics because some users particularly value the interpersonal relationship aspects of service interaction, while others acknowledge the increased efficiency and economic feasibility following the introduction of technology to the service context $[17,28]$.

Further, users, who are highly focused on the outcome of the interaction (characterized by a more objective orientation in their thoughts and behavior), tend to be more open towards service technology, whereas emotionally oriented users favor interactions with human staff [30].

To sum up, users' preference for interaction with chatbots vs. human staff seems to depend on the following characteristics amongst others [7,30]:

- Preference for chatbot interaction: rational (economic efficiency), optimistic, innovative, and technologically ready customers

- Preference for interaction with human staff: emotional, dependent on personal recommendations, and technologically anxious customers.

When focusing on the differentiation between rational vs. emotional personalities, the importance of considering the interaction with the situation becomes even clearer. At the beginning of the user journey, users are attracted by emotional aspects, whereas they pay more attention to rational/functional aspects later because the novelty effect wears off [6]. Addressing emotional needs through interaction with human staff may therefore be important in the early stages of the customer relationship, whilst chatbots may be beneficial to deal with purely rational inquiries later on.

Overall, from our literature review, customer implications with the use of AI have not been thoroughly investigated in the academic literature. Therefore, it is unclear how well a chatbot is capable of performing in customer interface and what kind of implications does a chatbot's possible failure have on user satisfaction or customers' continued willingness to use AI service technology. If companies justify the implementation of technology only with cost-savings and neglect the importance of user experience, net present value of investments may stay negative as unhappy customers switch service providers [28]. 


\section{The Case of the Chatbot EMMA}

\subsection{Implementation of $E M M A$}

Chatbots are used in different industry sectors. To better understand how they affect user experience, we look at the case of Emma, i.e., an existing chatbot that handles interactions between If, an insurance company, and its customers. Emma can be found on the company's website, where customers can use the "Ask Emma" function to interact with the chatbot. After having activated the feature, a chat window appears, in which customers communicate with the AI.

Emma is based on AI and machine learning and has been developed by the Finnish startup company GetJenny, to support companies' customer service and help desk operations. Emma enables the company to provide basic customer service in real-time 24-hours a day. Figure 2 illustrates the basic operations of Emma. In the course of the interaction, users are asked whether the chatbot provided the correct answer. If not, users are asked to rephrase their question and/or leave their contact details so that a human employee of the company can contact the user in due course. Hence, if Emma fails in managing the user inquiry, a human representative will take over.

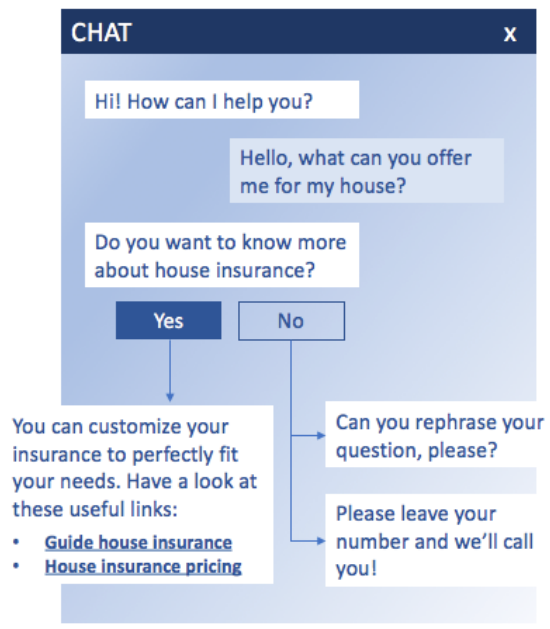

Figure 2: An Example of an Interaction Scenario with the Chatbot Emma. A User Can Start a Conversation with Emma by Clicking "Ask Emma" on the Company Website. When a User Starts a Conversation, Emma Tells the User that She Is a Customer Service Bot.

The joint project of chatbot Emma between GetJenny and If was launched in March 2017, with the aim of handling $10-20 \%$ of customer inquiries independently. At first, Emma was trained to answers questions of 50 of the most frequently asked topics. After six months of its launch, Emma is told to handle over $60 \%$ of all If's customer service interactions and is capable of answering questions of over 250 topics.

The fact that Emma is used by an insurance company illustrates that chatbots are also applied to deal with sensible topics. As this chatbot is used by an insurance company, which continuously excels in customer service, Emma needs to offer human-like interaction not to negatively affect the company's current high service ratings.

\subsection{User Study with $E M M A$}

Data collection. Following the introduction of the chatbot Emma, we examined the case of its application in order to identify how customers' preference for interaction with a chatbot is formed based on customers' quality perception and the consideration of personsituation interaction as outlined above. Specifically, we asked users after their interaction with Emma on the website of the insurance company to participate in a short survey about their experience with Emma. We asked about the following aspects:

a) problem-solving ability ("Was Emma able to solve your problem?")

b) overall satisfaction ("What is your overall satisfaction with the service provided by Emma?")

c) perceived technical quality (see Table 2 )

d) perceived process quality (see Table 2)

e) AI preference ("I would rather use Emma for customer service than a human."),

f) continuous usage ("I would use Emma again.").

We also asked demographic information (age, gender) and duration of customer relationship with the insurance company. For statements b-f, the respondents were given a 7-point Likert scale ranging from 'Strongly disagree' ('Highly dissatisfied' for b) to 'Strongly agree' ('Highly satisfied' for b). For the problemsolving ability statement ("Was Emma able to solve your problem?"), they had the following options:

- "Yes, Emma solved my problem entirely."

- "Partially. Emma could answer some of my questions but could not completely solve my problem."

- "No, Emma was not able to solve my problem and I was directed to a human.")

Since electronic surveys typically have a low response rate, users were encouraged to respond by raffling an Apple iPad tablet computer between the respondents. In our study, the response rate of the people who opened the questionnaire was $56.5 \%$. When inspecting the quality of the responses, 29 respondents had to be discarded because their responses were identical (for example, a line full of response options 1 or 7), or their response time was more than three times faster than the average response time. 
Table 2: Measurement Items for Technical Quality [21] and Process Quality [1]. The Observed Cronbach's Alphas (a) Indicate High Internal Consistency of Factors.

\begin{tabular}{|l|l|}
\hline \multicolumn{1}{|c|}{$\begin{array}{c}\text { Technical quality } \\
(\boldsymbol{a}=\mathbf{0 . 9 2 1})\end{array}$} & \multicolumn{1}{|c|}{ Process quality (a=0.929) } \\
\hline $\begin{array}{l}\text { - Emma was able to solve } \\
\text { my problem fluently. } \\
\text { - Emma didn't make } \\
\text { mistakes in my opinion. } \\
\text { - Emma was quick to solve } \\
\text { my problem. }\end{array}$ & $\begin{array}{l}\text { - Chatting with Emma was } \\
\text { fun. Chatting with Emma was } \\
\text { interesting. } \\
\text { - Emma gave me all the } \\
\text { information I needed. } \\
\text { makes it possible to get } \\
\text { customer service regardless } \\
\text { of time and place. } \\
\text { - Chatting with Emma } \\
\text { makes life easier. } \\
\text { - Chatting with Emma felt } \\
\text { good. } \\
\text { - Chatting with Emma } \\
\text { saves time. }\end{array}$ \\
\hline
\end{tabular}

We received a total of 225 usable responses from the users who had interacted with Emma. Of these, $86.7 \%(\mathrm{~N}=195)$ were existing customers of If and had typically been customers for more than five years. Around half $(52.9 \%, \mathrm{~N}=119)$ were male. The average age range for respondents was $40-49$, with $62.7 \%$ $(\mathrm{N}=141)$ of the respondents being over 40 years old.

The users could initiate a conversation with Emma on either the If website or a private customer page. In total, $44.0 \%(\mathrm{~N}=99)$ of the respondents were interacted on the website and $56.0 \%(\mathrm{~N}=126)$ on their private pages. After the conversation, the user has the option to participate in a survey by clicking a link leading to it. This allows the respondent to be transferred to the electronic survey, after which they can complete it. All the participants were aware of interacting with a chatbot, as this was specifically mentioned upon opening the chat dialogue box.

Results. In total, $38.2 \%(\mathrm{~N}=86)$ of the respondents reported of receiving a complete solution from Emma, without the need to direct the question forward to a human customer servant. On the other hand, $31.6 \%$ $(\mathrm{N}=71)$, answered that Emma had partial problemsolving skills, meaning that Emma was able to answer some of their questions but could not handle all of them, thus forwarding the customer to a human customer servant. Around the same number of customers $(31.6 \%$, $\mathrm{N}=59$ ) answered that Emma's problem-solving skills were deficient, meaning that Emma could not answer any of the customer's requests and forwarded the customer to a human customer servant. These results indicate satisfactory ability to solve the customers' problems, as Emma could at least partially solve $70 \%$ of the cases.

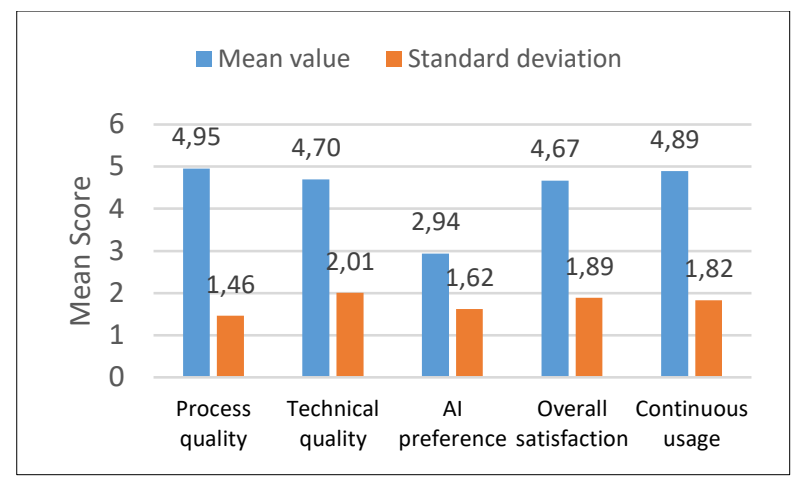

Figure 3: Mean Scores of Key Indicators on a 7-Point Scale.

As shown in Figure 3, the values of process quality $(\mathrm{M}=4.95)$ are slightly higher than the values of technical quality $(\mathrm{M}=4.70)$, although the difference is slight. Thus, the process quality ("soft aspect") can be considered slightly higher than chatbot Emma's ability to solve problems quickly, correctly, and smoothly. This is an interesting finding, as it implies Emma is not perceived as "cold" but rather as polite or friendly. The high standard deviation of technical quality $(\mathrm{SD}=2.0)$ relative to process quality $(\mathrm{SD}=1.46)$ is also noteworthy; it indicates Emma's ability to provide technical solutions is more varied than its way of communicating in a friendly manner.

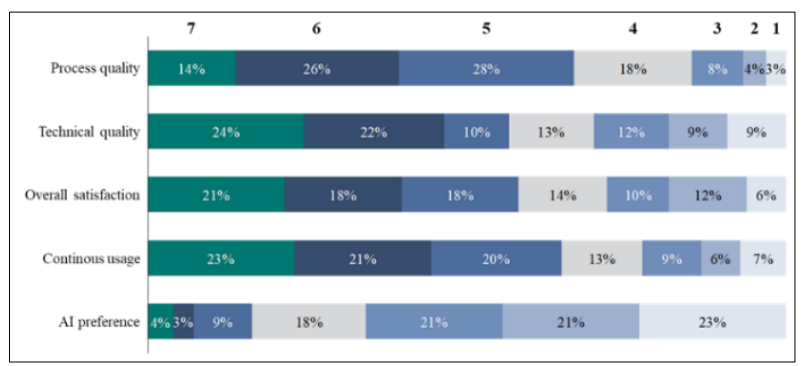

Figure 4: Frequency Distribution of the Key Indicators. The Horizontal Axis Has a Scale of 1 to 7, and the Vertical Axis Shows the Key Indicators.

As seen from Figure 4, more than $2 / 3$ of the respondents $(65 \%)$ have a low AI preference, with scores from 1 to 3 , which indicates that customers are currently not prepared to prefer AI customer service over a human. This proposition is further enhanced by the fact that AI preference had the lowest mean score of the measured variables $(M=2.94)$ (see Figure 3).

There is an interesting feature between technical quality and process quality: the mean for process quality is 4.9 , higher than 4.7 of the technical quality; yet, only $14 \%$ give process quality the highest score $(7 / 7)$ while the same number for technical quality is $24 \%$. Both overall satisfaction and continuous usage have a considerably higher number of positive responses, with 
scores of 5 to 7, making circa (ca.) $60 \%$ of the responses and scores of 1 to 3 , ca. $25 \%$. Again, these results indicate the positive user experience that the respondents generally had with Emma.

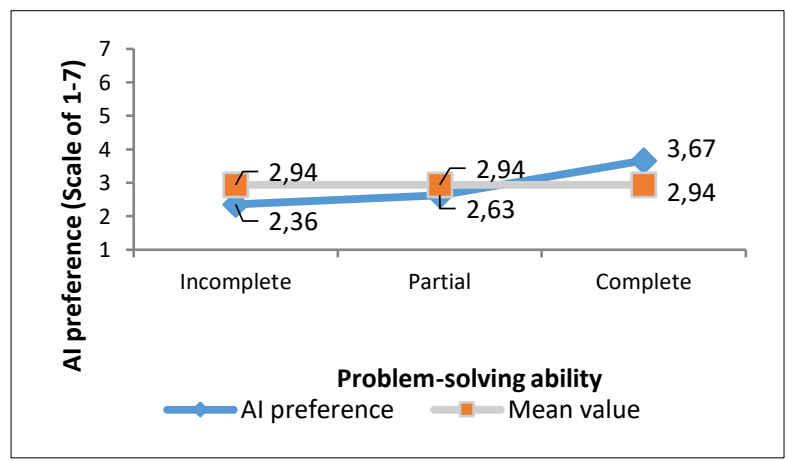

Figure 5: The Ratio of Chatbot's Problem-solving Ability and Artificial Intelligence Preference. As Emma's Problem-Solving Improves, Users' AI Preference Increases.

Other interesting findings include:

- There is a positive relation between Emma's problem-solving ability and users' AI preference (Figure 5) - this implies that users are more willing to use a chatbot if it can solve their problems, and vice versa.

- The correlation between overall satisfaction and Emma's problem-solving ability is weak to medium $\left(\mathrm{r}=34.4 \%, \mathrm{R}^{2}=11.2 \%\right)$, implying that satisfaction is not only related to the chatbot's ability to solve problems, but also to how these problems are solved.

Furthermore, there is an interesting interplay between technical and process quality (see Figure 6). Overall satisfaction is influenced more by technical quality $\left(\mathrm{R}^{2}=0.713 ; \mathrm{p}<0.001\right)$ than process quality $\left(\mathrm{R}^{2}=0.269 ; \mathrm{p}<0.001\right)$. For AI preference, the reverse applies - process quality is a much more impactful factor $\left(\mathrm{R}^{2}=0.478 ; \mathrm{p}<0.001\right)$ than technical quality $\left(\mathrm{R}^{2}=0.118 ; \mathrm{p}=0.052\right)$. These results indicate that while, without the functional 'intelligence' of a chatbot, the user is not satisfied, the functional intelligence alone is not enough for customers to prefer a chatbot. A pleasurable interaction experience is also required.

We also predicted AI preference with technical quality and process quality as independent variables. Regression analysis shows that technical quality is a significant predictor for $A I$ preference (Beta $=0.35$; tvalue $=5.03 ; \mathrm{p}<0.01)$ but does not explain much of the variance of $A I$ preference $(\mathrm{R} 2=0.170)$. When including process quality as a variable in the model, the impact of technical quality decreases to non-significant at 0.05 significance level (Beta $=0.14 ; \mathrm{t}$-value $=1.86 ; \mathrm{p}<0.1)$. In this model, process quality is more impactful
$($ Beta $=0.42 ; \mathrm{t}$-value $=5.30 ; \mathrm{p}<0.01)$ and adds to the overall explanatory power $(\mathrm{R} 2=0.272)$.

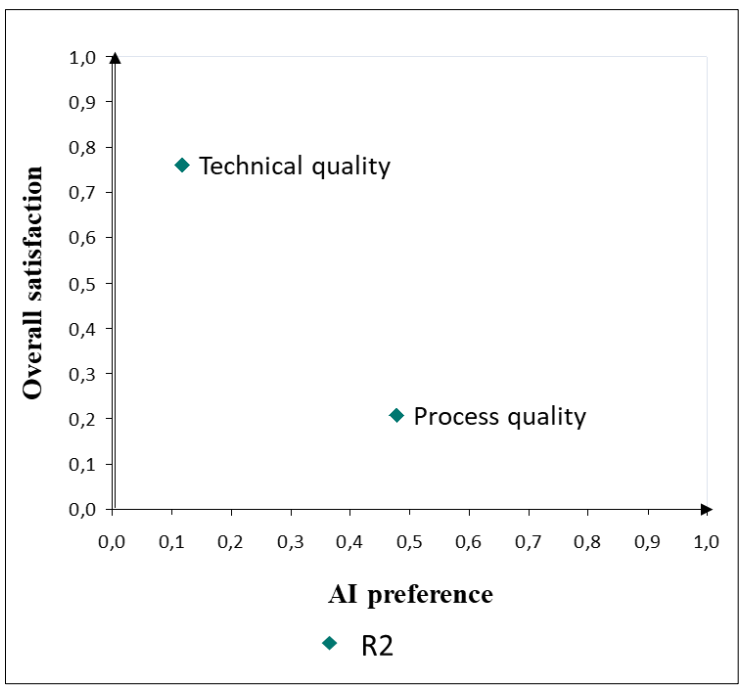

Figure 6: The R2's of Process Quality and Technical Quality to Overall Satisfaction and AI Preference.

However, it is counter-intuitive that technical quality "would not matter", as users are primarily using chatbots to solve their real customer service problems. Therefore, we decided to test the mediation effect, specifically if process quality mediates technical quality when predicting AI preference.

Mediation occurs if the direct relationship between technical quality and AI preference is significant (condition 1=TRUE), this relationship becomes insignificant as the mediator variable (i.e., process quality) is included (condition $2=T R U E$ ), and technical quality explains process quality (condition 3 ), which we also found to be the case (Beta $=0.58$; $\mathrm{t}$-value $=5.03$; $\mathrm{p}<0.001 ; \mathrm{R} 2=0.33)$. All the conditions of mediation are thus fulfilled, so it can be interpreted that process quality functions as a mediator between technical quality and AI preference.

Finally, neither customership $(\mathrm{p}=0.40)$, its duration $(p=0.17)$, gender $(p=0.94)$, or age $(p=0.90)$ had significant mean differences concerning AI preference.

\section{Discussion}

\subsection{What do the Results Mean?}

In the case of Emma, users seemed to be rather satisfied with the interaction and also indicated that they would be willing to use Emma again, although only a third of the users stated that Emma had dealt with their inquiry satisfactorily, whilst another third reported partial 
problems and the remaining stated that Emma did not offer any adequate responses.

The research shows that AI preference can be explained with quality that divides into two dimensions - technical quality and process quality, previously presented in the literature of service quality (see [11, 12]). The first dimension relates in AI context directly to the efficiency of the chatbot; that is, its ability to solve customer's problems. On the other hand, the process quality is associated with aspects of user experience, such as usefulness, convenience, accessibility, easiness, and speed of the interaction.

Results suggest that before the technical quality is right, there is no point in building the process quality, as the process quality strongly mediates the effect of technical quality on AI preference. This link is justified by the fact that process quality became the most important factor for the preference of $\mathrm{AI}$ in the analysis when accounting for both quality types, while technical quality is an antecedent for the process quality.

AI preference is formed so that the process quality explains the preference of chatbot and technical quality, in turn, explains the process quality. This implies that the priority enhancing a preference for artificial intelligence is to establish satisfactory problem-solving skills ("hygiene factor"), after which a pleasant user experience should be built (a "wow/competitive factor") for users to be ready to prefer the chatbot. Without technical quality, it is impossible to build AI preference, but this preference cannot also be built with technical quality. Ultimately, process quality is the element that allows customers to choose the chatbot instead of a living person.

Grönroos [11, 12] suggests the quality that the customer experiences consist of both technical and process quality in the traditional interaction between people. This study implies that the exact same conceptual elements of quality also determine the quality in the interaction between humans and machines. The conceptual elements of quality literature would, therefore, be the same in both artificial intelligence and humane customer service. However, decisive differences are likely to be seen when examining which of the concrete elements of process quality (empathy, enjoyability, accessibility, etc.) form the best possible artificial intelligence experience, or which of these quality elements users value the most. For example, further studies of service design can help to concretize the creation of superior AI customer experiences.

\subsection{Implications for Organizations}

Given our results, organizations are advised to ensure that chatbots meet technical and process quality requirements if they want to create preferences for chatbot interaction.

We want to highlight that customers might be willing to use chatbots and that they are more or less satisfied by the interaction; the preference for communicating with chatbots instead of human representatives of a company is not reality (yet). However, we identify certain drivers of the preference for chatbot interaction, which may help to stimulate such preference in the industry. For now, allowing customers to choose between the interaction with human staff or a chatbot appears as the most promising strategy. Some users may then function as early adopters, who can further promote the chatbot interaction.

Altogether, our case leads to the framework presented in Figure 7, which summarizes important drivers of customers' preference for interaction with a chatbot. This understanding will help organizations decide whether chatbots may be a useful tool for them and/or to guide organizations to successfully implement them.

Further, organizations need to be aware that not the pure technical features of AI are essential in creating customers' preferences for interacting with AI instead of human employees; customers' perceived process quality, as well as the usefulness of AI depending on the person-situation composition, need to be equally considered. Of course, chatbots should be in a position to adequately respond to customers' inquiries.

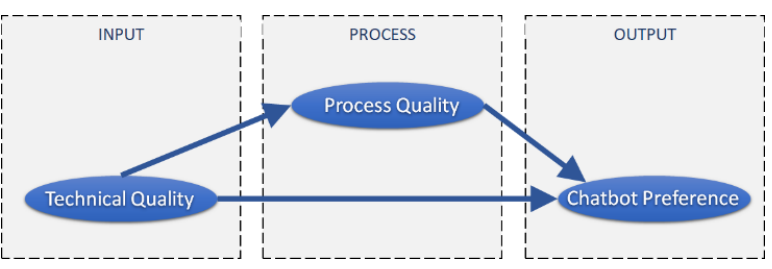

Figure 7: Framework Describing the Drivers of Customers' Preference for Using Chatbots.

As service failures need to be minimized in any service context, the technical quality of chatbots represents a hygiene factor, which customers expect in any service encounter. Building on this, customers' process quality perceptions may act as motivators, which can ultimately shape preferences for interaction with a chatbot. Process quality helps to ensure an enjoyable and customer-beneficial experience. Amongst others, process quality perceptions may improve if chatbots better simulate human-like interaction, so the specific dynamics of human interaction also become alive in interactions with a chatbot. This may include more emotional and targeted communication. If a chatbot achieves this, and we also add its specific strengths (e.g., immediate response, 24- 
hour availability), user preference for chatbot interactions seems to be more than just an illusion.

We want to draw attention to the necessity to carefully analyze and consider situational and customer characteristics. The implementation of chatbots, but the introduction of $\mathrm{AI}$, in general, requires a detailed examination of the context. Only if the context is sufficiently understood can measures be undertaken to facilitate the preference for interaction with a chatbot. For example, if the first interaction of a customer with a chatbot concerns a rather complex task, which the chatbot fails to solve, the customer can build up a history of negative experience, which will make further chatbot utilizations less likely.

Additionally, organizations need to decide whether they inform their customers that they are interacting with a chatbot. In practice, customers are sometimes exposed to chatbot interaction without knowing they are communicating with AI instead of a human representative. In such a context, ethical issues arise-for a full overview on ethical issues, see [36]. If customers accidentally find out that they are interacting with AI, negative consequences may emerge, particularly for loyal customers who might feel less valued. Further, as chatbots still frequently create service failures, organizations need to consider how customers attribute such service failure depending on whether they know that they are interacting with AI or not. While, in general, customers seem to be more forgiving if the service failure emerges from AI instead of human staff [28], solving real problems is essential for the success of chatbots in customer service.

For chatbot designers and managers, this means that having AI to solve their questions efficiently and correctly is not enough for customers, but the chatbot must be able to create enjoyable, pleasing, and useful experiences. This has important implications for organizations managing AI customer experiences and for artificial intelligence system vendors and developers, in developing the operational quality and allocating resources to increase the empathy, enjoyment, and pleasantness of their systems. However, it should be noted that the technical quality of AI, i.e., the functionality, must be satisfied before the elements of experimental quality are built. This is very natural since customer service is primarily contacted when seeking a solution for a specific problem.

\section{Conclusion}

Following digital transformation and disruption, organizations increasingly move towards AI-based automation to streamline their operations and to create better value for users and customers. The rapidness of such developments creates an inescapable urgency to develop and implement AI applications. However, there is limited research on users' interactions with AI in service scenarios, particularly distinguishing perceptions of technical vs. process aspects of AI. As today's technologically influenced users seek not just solutions for problems, but it also enhanced experiences, understanding how process quality, as well as technical quality, affects customer service encounters is an important step towards creating better customer value. Focusing on customer and non-customer interactions with an intelligent chatbot developed by a Finnish startup firm, our research shows how technical quality influences preference for AI interaction through increased process quality.

Findings offer interesting insights for service organizations at the application in end of AI, including that process quality (more concerned with user experience) is an important mediator for technical aspects such as problem-solving. Such insights are also useful for improving AI applications, since understanding contextual factors in the early stages of technological developments is a key step in their developmental process. Most importantly, the considered case also demonstrates that AI can be successfully implemented to support human workforce, as opposed to replace human employees; chatbots have the potential to handle first user inquiries followed by directing these users to the relevant organizational department operated by human staff.

\section{Acknowledgments}

We would like to thank If, the Finnish insurance company, for collaborating with us on this research.

\section{References}

[1] Almquist, E. et al. 2016. The elements of value. Harvard Business Review. 94, 9 (2016), 47-53.

[2] Anderson, E.W. and Sullivan, M.W. 1993. The antecedents and consequences of customer satisfaction for firms. Marketing science. 12, 2 (1993), 125-143.

[3] Bettencourt, L.A. et al. 2013. The secret to true service innovation. Business Horizons. 56, 1 (2013), 13-22.

[4] Buell, R.W. et al. 2010. Are self-service customers satisfied or stuck? Production and Operations Management. 19, 6 (2010), 679-697.

[5] Collier, J.E. and Bienstock, C.C. 2006. Measuring service quality in e-retailing. Journal of service research. 8, 3 (2006), 260-275.

[6] Collier, J.E. and Kimes, S.E. 2013. Only if it is convenient: understanding how convenience influences self-service technology evaluation. Journal of Service Research. 16, 1 (2013), 39-51. 
[7] Dabholkar, P.A. 1996. Consumer evaluations of new technology-based self-service options: an investigation of alternative models of service quality. International Journal of research in Marketing. 13, 1 (1996), 29-51.

[8] Davenport, T.H. and Klahr, P. 1998. Managing customer support knowledge. California management review. 40, 3 (1998), 195-208.

[9] Dhebar, A. 2016. Bringing new high-technology products to market: Six perils awaiting marketers. Business Horizons. 59, 6 (2016), 713-722.

[10] Fornell, C. et al. 1996. The American customer satisfaction index: nature, purpose, and findings. Journal of marketing. 60, 4 (1996), 7-18.

[11] Grönroos, C. 1982. Strategic Management and Marketing in the Service Sector. Swedish School of Economics and Business Administration.

[12] Grönroos, C. 2001. The perceived service quality concept-a mistake? Managing Service Quality: An International Journal. 11, 3 (2001), 150-152.

[13] Hilton, T. and Hughes, T. 2013. Co-production and selfservice: The application of Service-Dominant Logic. Journal of Marketing Management. 29, 7-8 (2013), 861881.

[14] Huang, M.-H. and Rust, R.T. 2018. Artificial intelligence in service. Journal of Service Research. 21, 2 (2018), $155-172$.

[15] Huang, M.-H. and Rust, R.T. 2013. IT-related service: A multidisciplinary perspective. Journal of Service Research. 16, 3 (2013), 251-258.

[16] Jarrahi, M.H. 2018. Artificial intelligence and the future of work: human-AI symbiosis in organizational decision making. Business Horizons. 61, 4 (2018), 577-586.

[17] Kahle, L.R. 1984. Attitudes and social adaptation: a person-situation interaction approach. Pergamon Press.

[18] Kaplan, A. and Haenlein, M. 2019. Siri, Siri, in my hand: Who's the fairest in the land? On the interpretations, illustrations, and implications of artificial intelligence. Business Horizons. 62, 1 (2019), 15-25.

[19] Ko, M.-C. and Lin, Z.-H. 2018. CardBot: A Chatbot for Business Card Management. Proceedings of the 23rd International Conference on Intelligent User Interfaces Companion (2018), 5.

[20] Kumar, A. and Telang, R. 2012. Does the web reduce customer service cost? Empirical evidence from a call center. Information Systems Research. 23, 3-part-1 (2012), 721-737.

[21] Lin, J.-S.C. and Hsieh, P.-L. 2011. Assessing the selfservice technology encounters: development and validation of SSTQUAL scale. Journal of retailing. 87, 2 (2011), 194-206.

[22] Marzocchi, G.L. and Zammit, A. 2006. Self-scanning technologies in retail: determinants of adoption. The Service Industries Journal. 26, 6 (2006), 651-669.

[23] Mustak, M. et al. 2021. Artificial intelligence in marketing: Topic modeling, scientometric analysis, and research agenda. Journal of Business Research. 124, (Jan. 2021), 389-404. DOI:https://doi.org/10.1016/j.jbusres.2020.10.044.

[24] Osburg, V.-S. et al. 2017. Unveiling ethical product features: The importance of an elaborated information presentation. Journal of cleaner production. 162, (2017), 1582-1591.

[25] Parasuraman, A. et al. 1988. Servqual: A multiple-item scale for measuring consumer perc. Journal of retailing. 64, 1 (1988), 12.

[26] Ravindran, D.S. 1970. An empirical study on service quality perceptions and continuance intention in mobile banking context in India. The Journal of Internet Banking and Commerce. 17, 1 (1970), 1-22.

[27] Ruane, E. et al. 2018. Botest: a framework to test the quality of conversational agents using divergent input examples. Proceedings of the 23rd International Conference on Intelligent User Interfaces Companion (2018), 64.

[28] Scherer, A. et al. 2015. The Value of Self-Service: LongTerm Effects of Technology-Based Self-Service Usage on Customer Retention. MIS quarterly. 39, 1 (2015).

[29] Shmueli-Scheuer, M. et al. 2018. Exploring the universe of egregious conversations in chatbots. Proceedings of the 23rd International Conference on Intelligent User Interfaces Companion (2018), 16.

[30] Simon, F. and Usunier, J.-C. 2007. Cognitive, demographic, and situational determinants of service customer preference for personnel-in-contact over selfservice technology. International Journal of Research in Marketing. 24, 2 (2007), 163-173.

[31] Venkatesh, V. 2000. Determinants of perceived ease of use: Integrating control, intrinsic motivation, and emotion into the technology acceptance model. Information systems research. 11, 4 (2000), 342-365.

[32] Venkatesh, V. and Davis, F.D. 2000. A theoretical extension of the technology acceptance model: Four longitudinal field studies. Management science. 46, 2 (2000), 186-204.

[33] de Visser, E.J. et al. 2016. Almost human: Anthropomorphism increases trust resilience in cognitive agents. Journal of Experimental Psychology: Applied. 22, 3 (2016), 331.

[34] Walker, R.H. et al. 2002. Technology-enabled service delivery: An investigation of reasons affecting customer adoption and rejection. International Journal of service Industry management. 13, 1 (2002), 91-106.

[35] Wang, D. et al. 2010. iHelp: An intelligent online helpdesk system. IEEE Transactions on Systems, Man, and Cybernetics, Part B (Cybernetics). 41, 1 (2010), 173-182.

[36] Wright, S.A. and Schultz, A.E. 2018. The rising tide of artificial intelligence and business automation: Developing an ethical framework. Business Horizons. 61, 6 (2018), 823-832.

[37] Zamora, J. 2017. Rise of the chatbots: Finding a place for artificial intelligence in India and US. Proceedings of the 22nd International Conference on Intelligent User Interfaces Companion (2017), 109-112.

[38] Zhou, M.X. et al. 2019. Getting virtually personal: chatbots who actively listen to you and infer your personality. Proceedings of the 24th International Conference on Intelligent User Interfaces: Companion (2019), 123-124. 